\title{
Das Desiderat der „angewandten oder empirischen Metaphysik“ - von der ANT zu Netzwerken sich selbst konditionierender Reflexionen?
}

\author{
Von Till Jansen und Werner Vogd
}

Zusammenfassung: Bruno Latour fordert zu recht für die Soziologie eine „empirische“ bzw. „angewandte Metaphysik“, um das Verhältnis von philosophischer bzw. metatheoretischer Begründung und sozialer Praxis vom Kopf auf die Füße stellen zu können. Allerdings fehlt ihm eine metatheoretische Konzeption, mittels der sich die Reflexionsverhältnisse einer empirischen Metaphysik konzeptionalisieren und rekonstruieren lassen. Im vorliegenden Artikel wird im Rekurs auf Gotthard Günthers Reflexionslogik eine Perspektive aufgezeigt, unter der sich das Latoursche Desiderat aufgreifen lässt. Auf diese Weise eröffnet sich der Weg zu einer empirischen Forschung, die weder in Hinblick auf gesellschafts- und differenzierungstheoretische Implikationen blind ist, noch essentialisierend die eigene Begriffsbildung mit der Logik der Praxis verwechselt.

\section{Einleitung}

Soziologische Theorie und qualitative empirische Forschung finden nicht leicht zueinander, wobei die Problemlagen unterschiedlich sind, abhängig davon, ob man sich von der Theorie kommend der Empirie nähert oder umgekehrt. ${ }^{1}$ Die Bewegung von der Theorie zur Empirie neigt dazu, sich in den von ihr selbst geschaffenen Begrifflichkeiten und Konzepten zu verfangen und diese mit der Realität zu verwechseln. Konzepte und Ausgangspunkte, die aus forschungspraktischer Sicht zunächst nützlich erscheinen, werden etwa essentialisiert, indem der Kontext vergessen wird, dem sich die Ausgangsunterscheidungen und die hieraus abgeleiteten theoretischen Begriffe verdanken. ${ }^{2}$ Empirische Daten und Protokolle werden dann nur noch subsumptionslogisch dem Baukastensystem des übergreifenden theoretischen Rahmens untergeordnet (etwa Jackson / Mazzei 2012; vgl. auch Keller 2014). Die Theorie übersieht, wenn man so möchte, die Empirie.

Die empirische Forschung hingegen neigt dazu, ihre eigene Metatheorie zu übersehen (Nassehi / Saake 2002). Während im Extremfall gar keine Metatheorie formuliert wird und die Ergebnisse der Forschung als reine grounded theory erscheinen (Glaser / Strauss 1999), tendieren die meisten Verfahren dazu, den Einfluss der eigenen Metatheorie auf die Ergebnisse zu vernachlässigen. Beispielsweise betreibt man in Folge eines essentialistischen Selbstmissverständnisses Biografieforschung, ohne ein Bewusstsein dafür zu entwickeln, dass nicht jeder Mensch in jeder Situation eine Biografie im Sinne des westlichen Individualismus braucht und hervorbringt (Knorr-Cetina 1989: 93). In gleichem Sinne ontologisiert man Konzepte wie ,Handlung' und ,Subjekt', wobei man den Umstand übergeht, dass diese Konzepte selbst erst in sozial und kulturell formatierten Zurechnungsprozessen entstehen. Oder man verwendet Kategorien gesellschaftlicher und funktionaler Differenzierung und

1 Auch wenn in den letzten Jahren vermehrt Versuche formuliert worden sind, hier wieder zu einem reflexiven Wechselspiel zu gelangen (siehe etwa Kalthoff et al. 2008; Lindemann 2002; Vogd 2011), bleibt dieser Befund gültig.

2 So Poppers Kritik am Essentialismus (1994: 17 f).

Soziale Welt 65 (2014), S. $451-467$ 
vergisst dann mehr und mehr, dass diese Kategorien erst aus bestimmten Praxen erwachsen. ${ }^{3}$ Wie auch immer die metatheoretischen Referenzen gelagert sind - wir treffen auf Subjekte, Objekte, Interaktionen, Handelnde, Systeme, gesellschaftliche Funktionssysteme oder andere Konstrukte, die als letzter Halt der Reflexion mitgeführt werden, ohne zu berücksichtigen, dass es die Praxis ist, die solche - und damit immer auch mögliche andere - Reflexionsleistungen hervorbringt und entsprechend auch wieder unterlaufen kann. Die üblichen Formen, Sozialforschung theoretisch zu fundieren, bleiben dementsprechend bei einem ,halbierten Konstruktivismus' stehen (Woolgar / Pawluch 1985) und führen ontologische Annahmen in Bezug auf die verwendeten Kategorien ein (seien sie ,realistisch' oder ,idealistisch') - und dies möglicherweise wider besseren Wissens, um im wissenschaftlichen Diskurs operationsfähig zu bleiben. ${ }^{4}$

Wie lässt sich diese vertrackte Lagerung methodologisch einholen? Die bislang radikalste Antwort stammt von der Actor-Network-Theory (ANT), die sich aus den science studies entwickelte. Anstatt hochgradig theoretisch angereicherte Metatheorien wie die hinter ,Subjekten“ oder ,Biographien“ vorauszusetzen, wird es hier der Empirie überlassen, was Akteur ist und was nicht, wer oder was Moralität aufweist und was nur reines Ding ist. Dabei verfällt die ANT jedoch nicht einem naiven Empirismus, sondern verfügt mit der Idee des Akteur-Netzwerks über eine abstrakte Minimalontologie, die sich situativ empirisch füllen lässt - eine soziologische Infrasprache wird einer soziologischen Metasprache als Alternative gegenübergestellt (Latour 2005). Das Problem wird also gelöst, indem eine Empirie vor dem Hintergrund einer Theorie gebildet wird, die zwar ein abstraktes Koordinatensystem bereitstellt, dabei jedoch auf die Bestimmung von Gegenständen wie ,Biographie‘, ,Subjekt` oder ,Individuum‘ verzichtet.

Nichtsdestotrotz wirkt die ANT in merkwürdiger Weise unfertig. So verharren die Analysen häufig auf einer deskriptiven Ebene und die Interpretation scheint in vielen Fällen beliebig und wenig methodisch kontrolliert. Das Vorhaben, Akteurhaftigkeit situativ zu bestimmen, scheint unausgereift und häufig arbiträr (Holzinger 2013). Ebenso scheint die ANT blind zu sein für unterschiedliche Reflexionspositionen, die ihren Grund in gesellschaftlicher Differenzierung haben (Farías 2013).

Wir möchten im Folgenden die These entwickeln und begründen, dass das Grundproblem der ANT in einer zu sparsamen Metatheorie liegt, der das theoretische Handwerkszeug zur Unterscheidung von Akteuren und Dingen fehlt. Hiermit einhergehend möchten wir einen Vorschlag unterbreiten, der eine differenziertere Infrasprache bereitstellt (vgl. auch Jansen 2014; Jansen / von Schlippe / Vogd 2015; Jansen / Vogd 2013; Vogd 2014 a). Im Anschluss an Gotthard Günther (1976, 1979 a, 1979 b) und Walther Bühl (1969) möchten wir eine Metatheorie zur Diskussion stellen, die klar zwischen Reflektiertem und Reflektierendem zu unterscheiden vermag und die auf diese Weise sowohl zwischen unterschiedlichen gesell-

3 Siehe aus dieser Perspektive auch die Kritik an einer falsch verstandenen Differenzierungstheorie bei Münch (1996) und Knorr-Cetina (1992). Interessant ist die Antwort von Nassehi auf den in KnorrCetina (1992) geäußerten Vorwurf an die Systemtheorie, denn hier wird eine polykontexturale Perspektive eingefordert, die zugleich Differenzierung wie Entdifferenzierung denken kann: „,Daß Kommunikation innerhalb wissenschaftlicher Experimente durch eine ausschließliche oder auch nur vorwiegende Codierung der Wahrheitssemantik vermittelt sein soll, ist empirisch nicht rekonstruierbar.' (Knorr Cetina 1992: 412). Hier hat sie Recht und Unrecht zugleich - selbstverständlich ist dies nicht rekonstruierbar, schon weil wissenschaftliche Experimente in der Tat Praktiken in Organisationen sind, in den Handlungen / Kommunikationen ganz unterschiedlicher Natur stattfinden - rechtliche, politische, intime, karrieretechnische usw. Aber dass man das rekonstruieren kann, setzt doch voraus, dass es offensichtlich unterschiedliche Logiken sind, die hier aufeinandertreffen." (Nassehi 2011: 29).

4 So dann auch Glaser, der zu einer >realistischen` Epistemologie zurückkehrt (2002). 
schaftlichen Reflexionspositionen differenzieren kann als auch in der Lage ist, Akteure klar von Objekten zu unterscheiden.

\section{Die ANT und das Desiderat einer empirischen Metaphysik}

Insbesondere von der Ethnomethodologie haben wir gelernt, ${ }^{5}$ dass es fruchtbar sein kann, das Problem des Verstehens einzuklammern, und stattdessen die Aufmerksamkeit auf die Untersuchung der Alltagsmethodologien zu lenken, welche das Verstehen im Sinne eines ,Doing Culture‘ herstellen. Wir gelangen auf diese Weise zu einer Perspektive, die nicht mehr nach der letzten Bedeutung von Worten zu fragen, also die Indexikalität der Begriffe nicht mehr aufzuklären braucht, sondern können dagegen auf all die Praxen schauen, die es gestatten, mit Situationen auch dann zurechtzukommen, wenn man sie nicht wirklich verstehen kann.

Diesen Ansatz radikalisiert die ANT im Anschluss an die science studies, indem sie die Position einnimmt, nicht nur den Akteuren ihre Deutungshoheit zu überlassen, sondern auch die epistemische und ontologische Bestimmung von Entitäten und Akteuren in der Schwebe zu halten. Worum es sich letztendlich handelt, zeigt sich dann in der Empirie. So arbeitet die ANT mit ,epistemischen Dingen“ (Rheinberger 2006), die sich dadurch auszeichnen, dass sie in Hinblick auf ihren ontologischen Status nicht vollständig definiert sind, sondern gewissermaßen darauf warten, in bestimmten Kontexten mit dieser oder jener Charakteristik hervorzutreten. Epistemische Dinge treten also als semantische Operatoren auf, mittels derer sich situations-, standort- und beobachtungsabhängig unterschiedliche ontologische Status zuweisen lassen und über die der Prozess dementsprechend gleichsam metaphysisch offen gehalten werden kann.

Hiermit erscheint es als ein vielversprechender Gedanke, dass auch die Sozialwissenschaften in ihrer Forschungspraxis in Hinblick auf ihre Gegenstände mit undefinierten epistemischen und ontologischen Status arbeiten könnten. Sie bekämen es dann jedoch nicht nur mit ,epistemischen Objekten', sondern auch mit ,epistemischen Subjekten` zu tun, nämlich mit ,Handelnden', ,Systemen“ etc., die kontext- und situationsabhängig ausflaggen oder eben auch nicht.

Der Ansatz, den Latour in diesem Kontext vorschlägt, besteht darin, homolog zur Methodologisierung der Verstehensleistung in der Ethnomethodologie (Wie wird Verstehen hergestellt?) einen ebenso fluide und dynamisch gestalteten Prozess des `Ethnotheoretisierens die Praxis zu postulieren. Gemeint ist hiermit, eine methodologische Haltung einzunehmen, entsprechend der Sprecher, Akteure, Psychen, Kommunikationen, Systeme etc. (in all diese Begriffe fließt selbstredend auch schon Theorie ein) beständig daran arbeiten, Reflexionen als Praxen auszuflaggen, die als „fungierende Ontologien“ erscheinen (Fuchs 2004: 11).

Menschen stellen sich dann beispielsweise je nach Kontext und Situation als Subjekt oder Objekt dar, als autopoietisch geschlossene Monaden oder als kommunikativ offene und beeinflussbare Prozessstrukturen, als Handelnde oder Erleidende. In Hinblick auf ihre eigentheoretische Leistung operieren sie als Idealisten, Konstruktivisten oder Realisten, als Monisten oder Dualisten, als Materialisten oder Transzendentalisten. Ihre Praxen, so die empirische Wendung dieser Perspektive, erscheinen damit immer schon von Metaphysik durchsetzt und - insofern diese Praxen reflexiv werden - von Philosophie und Epistemologie.

Die Frage nach der Richtigkeit von epistemischen Haltungen und ontologischen Positionen, also nach der Geltung der in der jeweiligen Ethnotheorie zum Ausdruck kommenden metaphysischen Haltung, ist hiermit methodologisch einzuklammern. Sie ist nicht mehr von

5 Siehe etwa Garfinkel / Sacks (2004). 
außen durch Theorie oder Reflexion zu entscheiden, sondern ergibt sich allein durch das in der Empirie entfaltete Arrangement. Vor diesem Hintergrund stellt sich für den Sozialforscher mit Latour die forschungspraktische Frage, ,wie man die den Akteuren eigene Metaphysik erkunden kann“ bzw. wie sich eine ,angewandte oder empirische Metaphysik“ betreiben lässt (Latour 2007: 89), über die sich die Verkettungen pragmatisch funktionierender Reflexions- und Konstruktionsleistungen rekonstruieren lassen. Sozialforschung darf und kann aus dieser Perspektive also nicht mehr ohne Erkenntnistheorie und Ontologie gedacht werden.

Das bedeutet allerdings - und dies erscheint aus der Sicht des klassischen wissenschaftstheoretischen Verständnisses als eine zunächst verstörende Konsequenz -, dass es keine Position mehr geben kann (sozusagen einen Gottes-Augen-Standpunkt), von der aus epistemische und ontologische Richtigkeit beansprucht werden kann. Vielmehr zeigt sich empirisch, welchen epistemischen und ontologischen Figuren die Verhältnisse in ihren Konstruktionsleistungen folgen. Mit Latour kehrt sich damit das Verhältnis von Theorie und Praxis um, denn ihm zufolge beinhalten die alltäglichen Praxen selbst bereits all die Reflexionsleistungen, welche die philosophische Tradition im Rahmen ihrer Denkgeschichte als idealtypische Reflexionsformen herausgearbeitet hat:

„Wie könnten Feldforscher einer Hausfrau, einem Buchhalter, einem Pilger, einem Kriminellen, einer Sopransängerin zuhören und dem folgen, was diese zu sagen haben, hätten sie nicht Hegel, Aristoteles, Dewey oder Whitehead, um ihnen zu helfen? Haben diese Autoren nicht viel nützliche Arbeit geleistet, um zu klären, was ein Akteur, eine Existenzform, eine Entität sein kann? Das bedeutet nicht, daß Philosophen es besser wüßten, tiefer eindrängen, fundamentaler wären als Sozialwissenschaftler, noch bedeutet es, daß sie der Soziologie eine ,Grundlage“ lieferten oder eine ,Metatheorie“ bereitstellten. Aber wenn man die Sozialwissenschaften von den Reservoirs philosophischer Innovationen abschneidet, dann ist das ein sicheres Rezept dafür, daß niemand je die philosophischen Innovationen gewöhnlicher Akteure bemerken wird, die oft über die professioneller Philosophen hinausgehen. Noch schlimmer wird es, wenn die Sozialwissenschaftler sich nicht nur der Metaphysik enthalten, sondern es geradezu als ihre Pflicht betrachten, auf einer äußerst beschränkten Liste von Handlungsträgern zu bestehen, und die unendlich mannigfaltigen Produktionen der Akteure ständig in diesen rudimentären Wortschatz übersetzten. Akteure bevölkern die Welt mit sehr unterschiedlichen Existenzformen, während die Soziologen des Sozialen ihnen erklären, aus welchen Bausteinen die Welt ,wirklich“ besteht.“(Latour 2007: 91)

Latour verweist hier zunächst auf zwei in Hinblick auf ihr Erkenntnispotential wenig fruchtbare Wege, wie Theorie und Empirie innerhalb der Sozialwissenschaften zueinander ins Verhältnis gesetzt werden können. Der eine besteht darin, dem, was man für den Menschen hält, ${ }^{6}$ subsumptionslogisch eine bestimmte Metaphysik zu unterstellen. Paradebeispiel hierfür ist der methodologische Individualismus. Demnach definiert man ,den Menschen modelltheoretisch als zweckrationalen Akteur, um dann in Hinblick auf die ihm unterstellte invariante Präferenzstruktur ein unteilbares Individuum zu postulieren, mit dem sich in Forschungsprozessen erfolgreich rechnen lässt. Dies ist allerdings nicht erstaunlich, da sich aus jedem Modell, das auf definierten Modellvariablen beruht, hypothesentestende Prozeduren ableiten lassen. Gleichzeitig wird in diesem Ansatz die ganze Fülle von Beziehungen ausge-

6 Bewusst ist hier die Rede vom Menschen, jedoch nicht vom Menschen als einem bestimmten, sondern im Sinne von Foucault als einer „empirisch transzendentalen Dublette“ (Foucault 1999: 384), welche nur als eine unscharfe Wolke in einem Raum psychologischer, soziologischer und biologischer Bestimmungsversuche, die nicht zu einer Eindeutigkeit finden können, gefasst werden kann (Foucault 1999: 416ff). Oder anders herum: Der Mensch wohnt nicht nur in einer Ontologie. 
blendet, welche menschliches Verhalten und Erleben und die hieraus erwachsenden Präferenzen ausmacht und konditioniert.

Der zweite Irrweg besteht darin, sich grundsätzlich aller metaphysischer Kategorien zu enthalten. Auf den ersten Blick mag man hier zwar näher an den Phänomenen zu sein, indem man auf eine vorherige Zurichtung der erhobenen Daten durch methodologische und metatheoretische Kategorien verzichtet. Doch damit vergisst man, dass Menschen ihrerseits ontologische und epistemische Haltungen einnehmen, dass man es in sozialen Phänomenen also mit Sinn und Reflexion zu tun bekommt, dass Menschen also ihre eigenen Ontologien erschaffen - wobei hier im Plural zu sprechen ist - und dass zudem die eingenommenen epistemischen oder ontologischen Haltungen in der Praxis nicht von Dauer sind, sondern situativ wechseln können. Ein Arzt, der einen Patienten von der Notwendigkeit einer Therapie überzeugen möchte, mag ihm beispielsweise zunächst als einem verständnisfähigen Subjekt begegnen, ihn ein wenig später aber schon als psychiatrischen Fall objektivieren, da er aufgrund seiner Krankheit nicht zur Therapieeinsicht fähig ist.

Je nach Netzwerkkonstellation reflektiert der Akteur dualistisch, monistisch, materialistisch, idealistisch, transzendental, dialektisch, sieht sich als Leib, Körper, Geist oder Beziehungswesen, erzeugt sich als Differenz zu sich selbst oder zu anderen und das mal instrumentell in Hinblick auf die Nutzenmaximierung, mal kommunikativ oder gar in Hinblick auf die Frage, ob die eigene Seele moralisch Schaden nehmen könnte. Einer ins Extrem getriebenen metatheoretischen Enthaltsamkeit entgeht also, dass Menschen in „fungierenden Ontologien" leben (Fuchs 2004: 11), dass sie sowohl Welt als auch sich selbst erkennen und reflektieren. Man sieht mit dieser Brille nur, dass die Akteure tun, was sie tun, ohne die Reichweite dessen bewerten zu können.

Latours Anliegen, die Fragen der Metaphysik in die soziale Praxis rückzubinden, ist ebenso richtig wie notwendig. Es stellt sich jedoch die Frage, ob dieses Projekt auf Basis seiner Konzeption des Akteur-Netzwerks in metatheoretisch konsequenter Weise und in der Folge ebenso empirisch durchgeführt werden kann.

\section{Eine massive Ausweitung des Akteursbegriffs}

Erinnern wir uns zunächst an die Besonderheiten der Akteur-Netzwerk-Theorie: Das wirklich Erstaunliche an diesem Ansatz ist eine massive Ausweitung des Akteursbegriffs, die mit einer gleichzeitigen inhaltlichen Entleerung der Begriffe ,actor' und ,agency ${ }^{6}$ einhergeht. Akteur kann hier prinzipiell alles sein, angefangen bei einzelnen Menschen bis hin zu Mikroben (Latour 1988), Computern (Latour 1987), Gespenstern und Göttern (Latour 2007) oder Märkten und Trennwänden (Garcia-Parpet 2007). Was genau ein Akteur ist, bleibt dabei jedoch unbestimmt. Anders als in anderen Akteurtheorien wird der Begriff theoretisch nicht ex-ante definiert, sondern komplett in die Empirie zurückgeworfen: „For ANT, a theoretical statement could never determine the extent to which nonhumans act and the nature of this agency. This is and remains definitively so for the perspective, an empirical question [Hervorhebungen im Original]" (Sayes 2013: 142).

In ethnographischer Tradition wird die Akteurhaftigkeit von Menschen oder nichtmenschlichen Dingen empirisch als Assemblage rekonstruiert. Ein Akteur ist hier nicht mehr die Einzelperson (oder das einzelne Ding) aufgrund seiner Eigenschaften, seiner Intentionalität, Rationalität oder ähnliches. Stattdessen ist agency selbst das Resultat einer bestimmten Konstellation. Sie entsteht als Desiderat einer bestimmten empirischen Lagerung. "This is, in fact, suggested by the very notion of an actor-network: the assembling together of a network of variable ontologies of variable times, and of variable spaces. Any actor - which, of course, includes nonhuman actors - is seen as necessarily a part of a more or less structured 
network" (Sayes 2013: 140). Demnach entscheidet das empirisch zu rekonstruierende Akteur-Netzwerk über die Frage, wer oder was Akteur ist und wer oder was nur Ding (Sayes 2013).

Unsere These an dieser Stelle ist, dass das Vorhaben der ANT aufgrund einer nicht ausreichend komplexen Metatheorie letztlich auf halber Strecke stecken bleibt. Dies hat verschiedene Gründe.

Zum einen bleibt die ANT, wie auch die Ethnomethodologie, in der jeweiligen Situation verhaftet. Gerade weil sie so sehr betont, dass die Akteure wissen, was sie tun, und dass man dieses Wissen bitte nicht mit irgendeiner Form der Latenzunterstellung überformen möge, ist sie blind für größere gesellschaftliche Zusammenhänge. Fragen der Beziehung unterschiedlicher institutioneller Logiken (Friedland / Alford 1991) sowie Referenzen auf Webers Wertsphären und Luhmanns Funktionssysteme müssen deshalb ausgeblendet werden (Farías 2013). Wenn also Knorr-Cetina (1992) der Systemtheorie vorwirft, dass diese für die Gleichzeitigkeit unterschiedlicher gesellschaftlicher Rationalitäten in Organisationen blind sei, weil sie jede Organisation nur unter eine Funktionslogik subsummiere, so ließe sich hier entsprechend anmerken, dass die ANT für unterschiedliche Rationalitäten in Organisationen blind ist, weil sie nicht über die Situation hinausblicken kann. Die Bedeutung, die Latour philosophischen Theorien oder gesellschaftstheoretischen Überlegungen zumessen kann, besteht vor diesem Hintergrund nur noch in deren Funktion als Vergleichshorizont: Man sieht, dass eine Situation eben genau so ist wie bei Hegel besprochen oder dass eine Handlung wie von Dewey konzeptionalisiert funktioniert. Analog könnte man feststellen, dass sich die Situation nicht so verhält, wie von Luhmann angenommen oder von Weber postuliert wurde. Damit sieht man gewissermaßen die Besonderheit, die Ausnahme, ohne jedoch weitergehende Interpretationen anstellen zu können.

Ein noch viel grundlegenderes Problem ist eng mit diesem Problem verbunden: Latours Metatheorie erlaubt es nicht, zwischen einem Objekt und einem Subjekt zu unterscheiden. Mit dem Versuch, eine essentialistische Deutung des Akteursbegriffs zu umgehen, schüttet er gleichsam das Kind mit dem Bade aus, da agency bei ihm nur noch eine Eigenschaft einer Entität ist. Ein Mensch, ein Sicherheitsgurt, Gott haben bei ihm in derselben Art agency, wie etwa Bäume grün sein können oder Katzen weich sind. Die ANT - die deshalb ebenso gut Aktant-Rhizom-Ontologie heißen könnte (Latour 2006) - subsumiert also die Ontologien der einzelnen Akteure der übergreifenden Ontologie des einen Akteur-Netzwerks, innerhalb dessen der einzelne Akteur nur noch ein Baustein, ein beliebiges Ding ist, über den der große Leviathan des Netzwerks verfügt. Als logische Konsequenz dieses Ansatzes muss unklar bleiben, wann ein Akteur ein Akteur ist (Holzinger 2013: 49 f), da überhaupt keine brauchbaren Kriterien vorliegen, nach denen Dinge und Akteure letztlich unterschieden werden könnten.

Damit entgeht der ANT allerdings die Pointe des Akteursbegriffs, denn in Anlehnung an Heidegger (2006) unterscheidet sich ein Akteur gerade dadurch vom Ding, dass er seine eigene Ontologie produzieren kann.

Es ist also weniger problematisch, dass in der ANT Dinge zu Akteuren gemacht werden, die gar keine Akteure sind, sondern dass Akteure zu Dingen gemacht werden, die sie nicht sind. Der einzige Akteur, der hier dann noch erscheint, ist das Akteur-Netzwerk selbst.

In Folge bleibt auch die Empirie der ANT in merkwürdiger Weise begrenzt. Zum einen ragt sie nie über ihren eigenen Gegenstand hinaus. Das jeweilige Akteur-Netzwerk wird analysiert, ohne dass eine gesellschaftstheoretische Einbettung stattfinden würde oder überhaupt möglich wäre. Darüber hinaus bleibt eine hierdurch informierte Analyse rein deskriptiv. Aufgrund der mangelnden Konzeptualisierung unterschiedlicher Reflexionspositionen 
kann sie nur die eine Ontologie des jeweiligen Akteur-Netzwerks beschreiben. Die ANT rastet sozusagen in dem naiven Realismus der einen Sinn-Welt ein und übersieht dabei sämtliche weiteren Prozesse der Sinnstiftung und funktionalen Differenzierung (Farías 2013). ${ }^{7}$ Oder um es schärfer zu formulieren: Die ANT muss gerade deshalb dem Common Sense des jeweiligen Feldes aufsitzen, weil sie an die objektive Realität ihres Netzwerks glaubt.

Das übergreifende Problem der ANT ist also mithin der Mangel an einer metatheoretischen Konzeption, die mit einer Pluralität von Ontologien und epistemischen Perspektiven innerhalb der empirischen Verhältnisse arbeiten kann. Obwohl die Pluralität von Ontologien behauptet wird, kann die ANT in ethnomethodologischer Tradition nicht anders, als immer wieder in einer Ontologie einzurasten, in der ein Akteur - oder ein Aktant - eben nichts anderes mehr ist als ein on - ein Seiendes wie eben auch ein Baum oder ein Strauch.

\section{Gotthard Günthers Reflexionslogik}

Will man sich einer empirischen Metaphysik nähern, die nicht von vornherein auf eine ontologische Perspektive festgelegt ist, so bedarf es einer Metatheorie, die systematisch zwischen verschiedenen Ontologien zu unterscheiden und diese in Beziehung zu setzen vermag. Hier beginnt Gotthard Günther mit der Kritik an der klassischen, zweiwertigen Logik. Die klassische Logik, so Günther (1978: 10ff), kennt gewissermaßen nur eine Reflexionsperspektive, da sie lediglich den Unterschied zwischen ,wahr' und ,falsch' kennt. Wenn eine bestimmte Aussage wahr ist, dann gilt ihre Wahrheit für alle Subjekte. Weicht eine Aussage von dieser Wahrheit ab, dann ist sie notwendigerweise falsch. In diesem Sinne bietet die klassische Logik nur die Möglichkeit einer einzigen, für alle denkbaren Subjekte identisch zu begreifenden Ontologie, die durch das Prinzip ,Tertium non datur ' festgelegt wird. Abweichende Perspektiven sind in dieser Logik nur als wahre Aussagen über abweichende Perspektiven zu begreifen. Dieser Annahme entspricht die formale Unterscheidung von wahren (p) und unwahren $(\neg$ p) Aussagen. Die Menge der wahren Aussagen stellt die ,wahre Ontologie' dar, während alle abweichenden Aussagen, die weiterhin im Bereich der Reflexion auftreten, notwendigerweise ,falsch“ sind. Es gibt also eine wahre Ontologie und eine Unmenge falscher Reflexionen. Das Sein hat dementsprechend Vorrang vor dem Denken (Günther 1978: $12 \mathrm{f}$ ).

Um diese Perspektive zu transzendieren, schlägt Günther vor, das Verhältnis von Negation und Gegenstand umzudrehen und die Negation zunächst als eine Reflexion zu fassen. Hier folgt er Hegels (1999) Auffassung vom Nichts in der Logik. Der Bereich des Nichts ist demnach nicht einfach das ,Falsche“, sondern die Reflexion im Gegensatz zum Sein. Wir treffen hiermit also auf der einen Seite auf eine positivsprachlich beschreibbare Welt, in der sich eineindeutig bezeichnen lässt, was ist oder nicht ist (nämlich das, was wir sehen, wahrnehmen und begrifflich bezeichnen können), und auf der anderen Seite auf die nur negativsprachlich formulierbare Welt eines offenen, noch nicht entschiedenen Seins, das nicht positiv erreichbar ist, jedoch über die Reflexion in die Welt eintritt und hier einen Unterschied macht (Günther 2000; Baecker 2013: 150). Erst an dieser Stelle wird deutlich, dass es sich bei einer Ontologie um eine Ontologie handelt, also um einen schon immer im logos reflektierten und interpretierten Raum des Seins. Damit ist zunächst zwar die Reflexion eingeführt und die Ontologie als Ontologie sichtbar gemacht, doch auch an dieser Stelle gibt es vorerst

7 Hiermit einhergehend lässt sich auch Kritik an Latours überscharfer Ablehnung der Theorie der funktionalen Differenzierung äußern. Als Hybridlagerung beruht die Moderne für Latour nur auf einer scheinbaren Differenzierung (Latour 2002). Es lässt sich mit der metatheoretischen Konzeption von Latour nur schwer denken, dass es Vermischung in hybriden Praxen und Differenzierung in distinkte Sinnsphären geben kann. Siehe auch Lindemann (2008: 354 f). 
nur einen Raum der Reflexion, der einem Raum des Seins gegenübersteht. Eine Pluralität von Ontologien verlangt demgegenüber, auch verschiedene Seinshorizonte und Reflexionen miteinander in Beziehung setzen und relationieren zu können.

Hier setzten Günthers Entwürfe einer mehrwertigen Logik an. Zunächst entwirft er eine Stellenwertlogik (Günther 1976 c: 137ff) und baut diese später zu einer Logik der „Polykontexturalität“ aus (Günther 1979 a). Dabei besteht die grundlegende Überlegung darin, dass in der klassischen zweiwertigen Logik mit der Operation der Negation ein Schritt angelegt ist, der über die Zweiwertigkeit selbst hinausreicht: Durch die Axiomatik der klassischen Logik, den Satz der Identität, den Satz vom ausgeschlossenen Widerspruch und den Satz des ausgeschlossenen Dritten wird eine Umtauschrelation zwischen $p$ ( $p$ ist) und $\neg p$ (der Sachverhalt $p$ ist nicht) etabliert, in der beide Positionen durch die Differenz zur jeweils anderen bestimmt sind. Einzig der Negator bildet sich dabei selbst ab (Günther 1963: 26). Günther weist an dieser Stelle darauf hin, dass der Negator die Zweiwertigkeit allein schon dadurch transzendiert, dass er diese als Umtauschrelation der Werte überhaupt erst konstituiert. Innerhalb der klassischen Axiomatik wird die hiermit verbundene Reflexionsbeziehung selbst nicht thematisiert. Sie bleibt damit unbestimmt.

Der Negator bildet für Günther dementsprechend den Ausgangspunkt für den Aufbau einer mehrwertigen Logik. Er muss insofern als eine transjunktionale Operation erscheinen (Günther 1976a), als dass er die Einheit einer bestimmten Zweiwertigkeit als logischen Raum erst konstituiert. Dieser logische Raum wird von Günther mit dem Begriff ,Kontextur' bezeichnet. Indem der Blick auf diese Operation der Konstitution gerichtet wird, wird diese indessen gleichsam transzendiert: Zunächst wird die Kontextur als Zusammenhang zwischen Reflexion und Reflektiertem sichtbar. Dadurch, dass sie sichtbar wird, wird performativ die Öffnung einer weiteren Kontextur vollzogen. Die ursprüngliche Kontextur wird hier in eine neue Ontologie eingebettet, ohne jedoch in dieser vollkommen aufzugehen.

Die transjunktionale Operation ist in diesem Sinne der Hinweis auf die jeweilige Beobachterposition. Sie ermöglicht das Umschalten zwischen verschiedenen Kontexturen als unterschiedlichen Orten der Reflexion. Günther (1979 b: 288) führt hier als Beispiel die Suspendierung der Frage nach Recht und Unrecht durch die Frage der Zurechnungsfähigkeit an. In der Diskussion über die Recht- bzw. Unrechtmäßigkeit einer Tat kann darauf hingewiesen werden, dass der Handelnde überhaupt nicht zurechnungsfähig war. Die transjunktionale Operation besteht in diesem Fall darin, die Frage nach Recht und Unrecht als Kontextur zu markieren, indem die Voraussetzungen der Anwendung dieses logischen Raums in Frage gestellt werden. Dies ist jedoch erst dann möglich, wenn man eine weitere Kontextur, nämlich die Diskussion über Zurechnungsfähigkeit eröffnet. ${ }^{8}$

Mehrere Kontexturen können eine Verbundkontextur bilden, die als eine mehr oder weniger stabile Struktur erscheint (Günther 1979 b: 291ff). Diese Gebilde ähneln auf den ersten Blick dem, was in der Systemtheorie als >strukturell gekoppelte Systeme gefasst wird (vgl. etwa Luhmann 1997; auch Renn 2006: 99ff), bzw. dem, was in der ANT als ein ,AkteurNetzwerk' betrachtet wird. Während allerdings ein Akteur-Netzwerk eine einheitliche Ontologie aufweist, die agency verteilt und Akteure als Identitäten innerhalb dieses Akteur-Netzwerks begreift, besteht eine Verbundkontextur gerade im Arrangement von widersprüchlichen und inkommensurablen epistemischen Perspektiven. Sie wird also nicht als eine einheitliche ontologische Ordnung verstanden, sondern stellt sich als ein polyzentrisches epistemisches Gebilde dar: „Was Grund und was Begründetes ist, wird geregelt durch den Stand-

8 Gerade an diesem Beispiel sollte klar werden, dass Bühls (2000) Kritik an Luhmanns Rezeption Gotthard Günthers nicht trägt. Denn die Polykontexturalität baut gerade auf dem Konzept einer Vielheit zweiwertiger Kontexturen auf, die ineinander verschachtelt sind. 
ort der Begründung. Der Wechsel des Standortes regelt den Umtausch von Grund und Begründetem. Jeder Ort der Begründung ist in diesem Fundierungsspiel Grund und Begründetes zugleich. Orte sind untereinander weder gleich noch verschieden; sie sind in ihrer Vielheit voneinander geschieden. Die Ortschaft der Orte ist bar jeglicher Bestimmbarkeit" (Kaehr 1993: $170 \mathrm{f}$ ).

Eine Verbundkontextur erreicht ihre Stabilität nicht dadurch, dass sich verschiedene Entitäten wechselseitig zu einer stabilen übergreifenden Entität konditionieren, sondern dadurch, dass unterschiedliche Ontologien im Widerspruch zueinander stehen und dieser Widerspruch eine Bewegung in Gang setzt, die ihrerseits den Zusammenhang bildet. ${ }^{9}$ So würden etwa in Günthers Beispiel die Frage nach der Zurechnungsfähigkeit und die Frage nach Recht und Unrecht zueinander in Widerspruch treten, um hieraus eine soziale Praxis erwachsen zu lassen, die weder mit der einen noch mit der anderen Seite identisch ist, sondern die Nicht-Identität in den Mustern der Bewegung bearbeitet.

Eine Verbundkontextur besteht also folglich aus mindestens drei verschiedenen Kontexturen. Sie ist weder auf der einen noch auf der anderen Seite zu finden, sondern bewegt sich dazwischen und stellt, indem sie eine eigene Struktur ausprägt, aus dem Widerspruch Stabilität her. Sie erscheint als eine soziale Praxis, die den Widerspruch bearbeitet, indem sie dynamisch unterschiedliche Ontologien ausprägt und arrangiert.

Auf diese Weise können durch die polykontexturale Logik heterarchisch verteilte Selbstorganisationsprozesse paradoxiefrei, d.h. ohne interne logische Widersprüche - dafür aber mit Widersprüchen innerhalb der verschiedenen Logiken - beschrieben werden. „Was Operator an einem Ort, ist Operand an einem anderen Ort, und umgekehrt. Damit wird die Zirkularität der Selbstbezüglichkeit von Operator und Operand nach der Figur des Chiasmus über vier Orte verteilt. Die Zirkularität löst sich auf in einen chiastischen Mechanismus von Ordnungs- und Umtauschrelationen, in dem die zwei fundamentalen Zirkularitäten zwischen Operator und Operand im Spiel sind, ohne dabei die fundamentale Hierarchie zwischen Operator und Operand zu verletzen. [...] Umtausch- und Ordnungsrelationen, Hierarchie und Heterarchie der Operativität und Relationalität, fundieren sich gegenseitig." (Kaehr 1993: $171 \mathrm{f})$

\section{Der Blick auf die wechselseitige Konditionierung logischer Räume}

Ein solchermaßen theoretisch aufgeladener empirischer Blick richtet sich auf die wechselseitige Relationierung verschiedener logischer Räume. Was heißt dies konkret? Entfalten wir das bereits angedeutete Beispiel eines Arztes, der seinen Patienten von der Notwendigkeit einer Therapie überzeugen möchte: Erster mag Letzterem zunächst einen Therapievorschlag unterbreiten und das Für und Wider schildern. Mit Blick auf die Selektion von Information und Mitteilung mögen nun seitens des Patienten innerhalb des vorgestellten medizinischen Rationals die Argumente abgewogen werden. Darüber hinaus lässt sich jedoch der medizinische Rahmen selbst verwerfen und vermuten, dass es um ein anderes Thema geht, beispielsweise darum, mit der Behandlung Gewinn zu machen. Zum andern können auch auf Arztseite weitere Reflexionsperspektiven wirksam werden. So mag dieser berücksichtigen, dass der Patient und seine Angehörigen rechtliche Beschwerdemacht einbringen oder möglicherweise eine Kritik äußern können, die im Netzwerk der anderen professionellen Akteure (etwa der Einweiser) zu Folgereflexionen führt. Insgesamt wird es hier also zu einem komplexen Arrangement unterschiedlicher Reflexionen kommen, die sich auf andere Reflexionen beziehen und sich entsprechend konditionieren (Jansen / Vogd 2013). Da die medizinische Praxis, die rechtlich wirksame Dokumentation dieser Praxis, die Abrechnung

9 Hier werden wieder die hegelianischen Wurzeln Günthers deutlich. 
der Leistungen sowie die einzelnen Ebenen der ärztlichen Hierarchie nur lose miteinander gekoppelt sind, können unterschiedliche Lösungen gefunden werden. „So kann behandelt werden, ohne zu behandeln, Rechtmäßigkeit hergestellt werden, indem Unrechtmäßiges nicht dokumentiert wird, wirtschaftlich gearbeitet werden, indem Medizin vorgetäuscht wird, wo anderes stattfindet, um an anderer Stelle umso mehr (ansonsten nicht bezahlbare) Medizin stattfinden zu lassen." (Vogd 2007: 317) Darüber hinaus kann jedoch noch mehr geschehen. So kann möglicherweise der Blick auf die merkwürdige Dualität des Körpers als Leib/Körper gerückt werden (Plessner 2003). Während der Patient eben noch Leib war, wird der Körper plötzlich zu einer eigenen Kontextur, die eine Eigenständigkeit und Widerspenstigkeit aufweist, die erst interpretiert werden will. Umgekehrt kann die Leibhaftigkeit des Körpers plötzlich fraglich werden, wenn etwa unklar ist, ob ein Patient hirntot ist (Lindemann 2002).

Wie in den Akteur-Netzwerken einer ANT-Beschreibung rechnen wir hier also damit, dass auch das Recht, die Dokumentation (Berg 1996), die Wirtschaft, der Patient und anderes an der Ausgestaltung des Patienten- und Arztverhaltens beteiligt sind. Wir rechnen ebenfalls mit einer hybriden Struktur und lassen offen, wer oder was als Akteur erscheint oder nicht (Lindemann 1999). Im Unterschied zur ANT sind es allerdings nicht die positivsprachlich fassbaren Dinge, die zum Handeln antreiben, sondern Reflexionen, die durch andere Reflexionen konditioniert sind. Reflexionen sind jedoch - genauso wie Sinn - weder sichtbar noch in einer anderen Weise fassbar.

Die hier entwickelte Perspektive lässt sich auch auf Aktanten übertragen, denen üblicherweise der Status eines Dings zugeschrieben wird, man denke etwa an eine Zuckerkugel oder eine Oblate aus Weizenmehl. Das Beispiel der homöopathischen Pille oder der Hostie zeigt auf, dass auch diese Aktanten in andere epistemische und ontologische Konfigurationen treten können, wenn sie in entsprechende Reflexionsverhältnisse eingebettet sind. Wie in anderen Fällen lassen sich mit der ANT hier lediglich Assoziationen positivsprachlich bezeichenbarer Aktanten entdecken, z.B. ,Hostie $=$ Oplate + Gott` oder , homöopathisches Medikament $=$ Pille + Medizin'. Der ontologische Status von Abstrakta wie Medizin, Gott etc. kann dementsprechend nicht aus der Empirie abgeleitet werden, sondern muss durch das Homologiepostulat der ANT gesetzt werden.

Demgegenüber können die hiermit verbundenen Lagerungen mit Hilfe der Güntherschen Reflexionslogik in zweierlei Hinsicht differenzierter und Empirie-näher rekonstruiert werden.

Zum einen kann eine klarere Unterscheidung zwischen Reflexivität und Irreflexivität gezogen werden. Während agency in der ANT immer eine graduelle Angelegenheit ist (Sayes 2013) und häufig unklar bleibt, ob die Zuschreibung etwa dem Belieben des jeweiligen Autors entspringt (Holzinger 2013), lässt sich mit Günther recht klar identifizieren, ob ein neuer logischer Raum eröffnet wird und welche Stellung er im Gesamtarrangement hat. Hierbei wird zwischen drei verschiedenen Formen unterschieden (Günther 1976), die sich ohne Weiteres in der Praxis wiederfinden lassen (vgl. auch Jansen 2013): ${ }^{10}$

10 Der besondere Charme der hier vorgeschlagenen Perspektive besteht darin, dass letztlich nur wenige logische Operationen benötigt werden, um den metaphysischen Strukturreichtum sozialer Praxen aufzuschließen: (1) Die Negation, welche einen logischen Raum als Kontextur aufspannt, in der im Sinne einer zweiwertigen Logik eine Subjekt-Objekt-Dichotomie erscheint; (2) Die Reflexion, welche qua Zurechnung ein weiteres Subjekt konstituiert; (3) Die Rejektion als Reflexionsoperation, welche die Wahl einer Kontextur verwirft, um eine andere zu affirmieren; (4) Die Konditionierung bzw. Moderation des Arrangements von Kontexturen durch andere Kontexturen. 
Teilweise Rejektionen verweisen Eigenschaften, Dinge, Aussagen usw. in einen anderen Bereich, der nicht weiter bestimmt wird. So kann etwa die Aussage eines Assistenzarztes als Unfug verworfen werden.

Totale undifferenzierte Rejektionen hingegen verweisen eine Aussage, Eigenschaft, Praxis etc. in einen anderen Bereich, der benannt, jedoch in seiner Binnenstruktur nicht weiter reflektiert wird. Beispielsweise können neue Vorschriften zur Aktenpraxis einer unfähigen Verwaltung zugerechnet werden, welche die ärztliche Arbeit nicht versteht.

Totale differenzierte Rejektionen verweisen auf einen ebensolchen Bereich und reflektieren ihn zusätzlich in seiner Binnenstruktur. Hier kann beispielsweise die neue Aktenpraxis in ihrer Funktion für die Verwaltung verstanden werden und es können Techniken entwickelt werden, mit denen auf diese Ansprüche der Verwaltung eigegangen werden kann, ohne jedoch die Vorschriften voll umzusetzen.

Somit beginnt Agency in diesem Schema erst bei Punkt C. Erst wenn eine Kontextur als ein eigener, adressierbarer Weltbezug auftritt, der Wünsche, Launen, Interessen etc. hat, ${ }^{11}$ erscheint ein ,Du', das von einem ,Ich“ als Interaktionspartner begriffen werden kann - sei es nun eine Verwaltungsstelle, ein Patient oder die Ehefrau.

Zum anderen eröffnet die Günthersche Reflexionslogik die Möglichkeit, mehrere Ontologien gleichzeitig mitzuführen und situativ aufzurufen (Jansen 2014). Eine Ontologie entsteht bzw. verschwindet immer dann, wenn eine Kontextur abhängig von einer konkreten Reflexionspraxis geöffnet bzw. geschlossen wird. Die Einnahme einer Hostie oder eines homöopathischen Medikaments aus Perspektive einer göttlichen oder magischen Kraft zu reflektieren und entsprechend empfinden zu können, schließt nicht aus, denselben Sachverhalt ein wenig später auch als Aberglauben deuten zu können.

Agency wäre aus der vorgeschlagenen Perspektive als eine Reflexivität zu identifizieren, die allerdings nicht ex ante definiert werden kann und darf. So kann etwa eine Organisation als Reflexionsinstanz mit eigener Ontologie auftreten, ebenso können die Sicherheitsbestimmungen im Automobilbau als Akteur erscheinen oder Gott, aber auch Tabletten oder Figuren in einem Computerspiel, wenn diese in einen entsprechenden Reflexionszusammenhang eingebettet sind. Ob und wie dies geschieht, bleibt der Empirie überlassen - einer Empirie, die sehr klar zwischen Entitäten innerhalb einer Ontologie (z.B. dem Sicherheitsgurt oder einer Marienfigur) und der jeweiligen Ontologie selbst (dem logischen Raum der Sicherheitsbestimmungen bzw. der Reflexion transzendenter Kraft) unterscheiden kann (vgl. Latour 1989). So zeigt sich beispielweise bei einigen Computerspielern empirisch ein Wechseln bzw. Oszillieren zwischen temporären Personalitätszuschreibungen, wobei die computerbasierten Spielfiguren menschlich empfunden und behandelt werden, und dem vorrangigen Modus einer Trivialitätserwartung, die mit technisch vermittelten Programmen rechnen lässt. Die Pointe besteht dabei darin, dass empirisch unterschiedliche, nicht zu vereinbarende Ontologien nebeneinander auftreten: Der Spielende weiß, dass er es mit einer Maschine zu tun hat, fühlt und verhält sich teilweise dennoch, als ob er einem menschenähnlichen Wesen gegenüber stünde (Harth 2014: 232ff). Oder um nochmals das Beispiel der homöopathischen Medikamente aufzugreifen: Ein Ingenieur mag als Anhänger der Schulmedizin davon überzeugt sein, dass ein homöopathisches Mittel nichts beinhaltet, was wirksam sein könnte, was ihn aber letztlich nicht daran hindert, diese Präparate trotzdem einzunehmen, um in Folge Wirkeffekte zu spüren, welche dann in anschließender Reflexion der eigenen Psyche oder gar dem Placeboeffekt zugerechnet werden (Vogd 2011: 317ff).

11 Siehe zum Erscheinen der >Du<-Perspektive in der neurobiologischen Forschung Lindemann (2006). 
Die hiermit verbundenen Lagerungen können mit Hilfe der Güntherschen Reflexionslogik differenzierter und Empirie-näher rekonstruiert werden. Sie erscheinen vor diesem Hintergrund als sich wechselseitig konditionierende Reflexionen, wobei im Sinne der polykontexturalen Logik die Gleichzeitigkeit unterschiedlicher Reflexionsperspektiven eher die Regel als die Ausnahme darstellt. So wie der durch den Computer generierte Spielpartner in der zuvor zitierten Studie (Harth 2014) sowohl als technisch generierte triviale Programmstruktur auftritt als auch als eine Wesenheit, die den menschlichen Spieler emotional berühren kann, kennt die polykontexurale Beschreibung die Kopräsenz und Überlagerung multipler Ontologien. Sie kann das Gesetz vom ausgeschlossenen Dritten in Richtung eines Sowohlals-auch wenden, ohne dabei auf eine distinguierte Beschreibung der jeweils lokalen Reflexionsverhältnisse verzichten zu müssen.

Erst auf diese Weise lässt sich dem Desiderat einer empirischen Metaphysik gerecht werden, die in konkreten Verhältnissen nicht selten auf Lagerungen trifft, in denen unterschiedliche, sich widersprechende Ontologien nahe nebeneinander liegen und dementsprechende Paare aus divergierenden epistemischen und ontologischen Haltungen sich nicht ausschließen, sondern sich über verteilte Standorte wechselseitig konditionieren und instruieren können (Jansen 2014). Das Paradebeispiel hierfür ist die Frage der Subjektivität und das hiermit verbundene Problem des freien Willens, das sich bei genauer Betrachtung als Frage der Reflexion aus unterschiedlichen Perspektiven und der wechselseitigen Konditionierung dieser Perspektiven erweist. Ebenso können und dürfen auch Existenzaussagen wie ,Es gibt Gott', ,Es gibt keinen Gott', aber auch ,Es gibt Systeme', ,Es gibt Gesellschaft', ,Es gibt Biografien', ,Es gibt Akteur-Netzwerke' etc. radikal in die Empirie zurückverlegt werden. Hier liegen dann, wie schon Wittgenstein in seinen Studien über Gewissheit aufgezeigt hat, ${ }^{12}$ Ontologie und Epistemologie, Glaube und Wissen nahe und oftmals kaum trennbar nebeneinander. Aus der Perspektive polykontexturaler Reflexionsverhältnisse heißt dies nichts anderes, als dass ein diesbezügliches Einrasten in eine Gewissheit weiterer Reflexionen zur Stabilisierung bedarf, dass also das situative Einrasten in eine fungierende Ontologie (Fuchs 2004) nur im Kontext eines bodenlosen Netzwerks inferenzieller Beziehungen (Brandom 2000) vonstatten gehen kann, die sich wechselseitig konditionieren.

\section{Diskussion}

Ausgangspunkt unserer Argumentation war die Annahme, dass die Idee einer soziologischen Infrasprache, die der ANT zugrunde liegt, zurzeit das beste theoretische Angebot ist, um dem Dilemma von Theorie und Empirie in der qualitativen Forschung zu begegnen. Gleichsam leidet die ANT selbst an einer gewissen Schwammigkeit und Beliebigkeit in der Empirie und vermag deshalb nicht so recht über die reine Deskription ihres jeweiligen Gegenstandes hinauszugehen. Vor diesem Hintergrund eröffnet Günthers Konzeption der Polykontexturalität die Möglichkeit zu einer Protosoziologie, die hier sowohl eine hinreichende analytische Schärfe ermöglicht wie auch die Rückbindung an gesellschaftstheoretische Diskurse erlaubt, ohne aber dabei die Idee einer Infrasprache aufzugeben, mit der sich ,das Soziale flach halten“ lässt (Latour 2007: 286ff). Latours berechtigte Kritik am Soziologismus und Essentialismus gewinnt mit Günther ein reflexionslogisches Instrument, mit Hilfe dessen es gelingt, über ethnografische Deskriptionen, die letztlich einer Seinslogik verhaftet bleiben müssen, hinauszugelangen, ohne sich jedoch der Tiefenschärfe einer Analyse zu ent-

12 Ein schönes Beispiel mit Verweis auf kommunikative Konditionierung von Gewissheit: „Der Satz ,Ich kann mich nicht irren' wird sicher in der Praxis gebraucht. Man kann aber bezweifeln, ob er dann in ganz strengem Sinne zu verstehen ist, oder ob er eher eine Übertreibung ist, die vielleicht nur zum Zweck der Überredung gebraucht wird.“(Wittgenstein 1992: 255). 
äußern, die um die Prozesse der gesellschaftlichen und kulturellen Differenzierung als einer alltagspraktischen Reflexionsleistung weiß.

Die vorgeschlagene Perspektive bietet dabei nicht nur eine methodische Erweiterung der ANT, sondern erlaubt vielmehr den Brückenschlag zu Theorien gesellschaftlicher Differenzierung, ohne sich dabei in die Widersprüche zu verheddern, die aus zu einfachen Kombinationen resultieren (Farías 2013). Sie bietet darüber hinaus eine methodische Wendung dessen, was derzeit in systemtheoretischen Kreisen als ,Weltgesellschaft' diskutiert wird (vgl. Stichweh 2000: 16ff). Die Unterscheidungen zwischen Mikro, Meso und Makro verschwinden hier nämlich im Konkreten einer Lebenspraxis, für die nicht nur das Präsente, sondern auch das Apräsente - sei es zeitlich (in Form von Geschichte und Zukunft) oder räumlich (als Perspektive der Nicht-Anwesenden) - als Reflexion im Sein erscheint und hier als Information einen Unterschied macht. Die Weltgesellschaft - sofern sie in der Weise real ist, dass sie sich als Reflexion manifestiert - macht im Hier und Jetzt einen Unterschied, indem sie eine jeweils spezifische Konstellation von Identität und Nichtidentität, von Subjekt und Objekt, von Konkretion und Abstraktion moderiert. ${ }^{13}$ Entscheidend ist jeweils, wie der Prozess der Reflexion situativ seine eigene Metaphysik mitproduziert, also welche Reflexionsperspektiven sich im Arrangement selbst arrangierender Reflexionsperspektiven einspielen, um jeweils spezifische kulturelle Formen zu entwickeln, die ihrerseits bestimmte Perspektiven und Perspektivierungen als >real und andere als >irreal erscheinen lassen. ${ }^{14}$ Die Empirie zeigt sich dann als eine „Gesellschaft der Gegenwarten“ (Nassehi 2011), die darauf beruht, dass das, was an einem Ort geschieht, an einem anderen Ort mitreflektiert werden kann, und dass dies wiederum am ersten Ort reflektiert wird, und sei es nur in der Form, dass man zwar nicht weiß, was dort geschieht, es aber prinzipiell wissen könnte und zumindest dies berücksichtigend in die Reflexion mit eintritt, um dann das hieraus entstehende Arrangement mitzuprägen (Jansen / Vogd 2013).

Gleichzeitig werden hier Anschlüsse an eine theoretische Empirie in der Tradition der philosophischen Anthropologie möglich, die ebenfalls die Bestimmung dessen, was mit Subjekt, Subjektivität und Du-Perspektive gemeint ist, radikal der Empirie überlässt (Lindemann 2002; Vogd 2010). ${ }^{15}$

All dies eröffnet die Möglichkeit einer gesteigerten Rigidität in der empirischen Analyse, für die hiermit geschärfte theoretische Konzepte bereitgestellt werden sollen. Der Netzwerkgedanke der ANT wird damit einerseits beibehalten. Andererseits gelingt es uns aber mit dem oben Gesagten, den epistemischen und ontologischen Status der Aktanten konsequent in der Schwebe zu halten. Anstelle eines Netzwerks aus Dingen rekonstruieren wir ein Netzwerk aus Reflexionsverhältnissen, die dann Objekte und Subjekte ausflaggen lassen. Doch hierbei handelt es sich um epistemische Dinge und epistemische Subjekte, deren Status nicht

13 „Soziologisch gesprochen“ treffen wir hier mit Nassehi auf „eine Welt, in der gleichzeitig und bisweilen unabhängig voneinander unterschiedliche Beobachtungsverhältnisse statthaben, die sich schon aus empirischen Gründen nicht ignorieren können - oder besser: die sich paradoxerweise nur ignorieren können, wenn sie das aktiv tun. Wenn etwa eine ökonomische Sichtweise auf beliebiges Phänomen all das, was geschieht, explizit darauf eingrenzt, ob gezahlt werden soll oder nicht [...], dann sieht diese operative Perspektive zunächst nichts anderes - muss das aber in einer Gesellschaft durchsetzen, die das Problem auch politisch oder rechtlich moralisch beobachten könnte.“ (Nassehi 2011: 28).

14 Insbesondere Luhmann hat darauf hingewiesen, dass nicht nur das Vertraute, sondern auch das Unvertraute durch die Kommunikation real erscheinen kann, und sei es nur, dass man weiß, dass man es wissen könnte, wenn man nur wollen würde (vgl. Luhmann 1996: 280).

15 Gleiches gilt übrigens seit der Quantenmechanik auch für die Physik. Auch hier sind die Beobachterverhältnisse nur noch polykontextural, das heißt auf Basis einer mehrwertigen Logik zu konzipieren, vgl. Vogd (2014 b). 
von vornherein und für alle Reflexionsorte bestimmt ist, sondern nur in Hinblick auf jeweils einen spezifischen Knotenpunkt im Netzwerk der Reflexionen rekonstruiert werden kann (Jansen / von Schlippe / Vogd 2015).

Auf diese Weise sind wir weder genötigt, dass Subjekt überhöhend zu affirmieren noch zu negieren. Analysen vor dem Hintergrund einer mehrwertigen Logik eröffnen also eine Reihe von Brückenschlägen, die über die jeweilige konkrete Empirie hinausgehen. Die jeweilige Konstruktion von Subjektivität erscheint damit nicht nur in einem konkreten Hier und Jetzt, sondern kann im Rückgriff auf all die uns zur Verfügung stehenden Konzepte gesellschaftlicher Differenzierung interpretiert werden (Jansen 2014).

Mit dem vorliegenden Beitrag folgen wir Hirschauers (2008: 165) Plädoyer für eine „,Enthemmung“ der Beziehungen von Theoriebildung und qualitativer Forschung“ und möchten neue „Bindungseffekte“ (Hirschauer 2008: 165) sowohl auf Seiten der Theoriebildung wie auch der Empirie ermöglichen (vgl. auch Keller 2014). Denkbar wird diese Brücke zwischen Gesellschaftstheorie und Empirie jedoch erst durch eine hinreichend abstrakte metatheoretische Konzeption, die gerade deshalb in metaphysischen Fragen analytisch auflösungsfähig ist, weil sie diesbezüglich inhaltlich hinreichend unbestimmt bleibt. In diesem Sinne könnte, so unsere Vermutung, der Rekurs auf Gotthard Günther nicht nur theoretisch, sondern auch empirisch für die Sozialwissenschaften hilfreich sein (hierzu Jansen / von Schlippe / Vogd 2015).

\section{Literatur}

Baecker, Dirk (2013): Beobachter unter sich. Eine Kulturtheorie, Berlin.

Berg, Marc (1996): Practices of Reading and Writing: The Constitutive Role of the Patient Record in Medical Work, in: Sociology of Health \& Illness 18, S. 499-524.

Brandom, Robert B. (2000): Expressive Vernunft, Frankfurt / Main.

Bühl, Walter L. (1969): Das Ende der zweiwertigen Soziologie: Zur logischen Struktur der soziologischen Wandlungstheorien, in: Soziale Welt 20, S. 162-180.

Bühl, Walter L. (2000): Luhmanns Flucht in die Paradoxie, in: Peter-Ulrich Merz-Benz / Gerhard Wagner (Hrsg.), Die Logik der Systeme: Zur Kritik der systemtheoretischen Systemtheorie von Niklas Luhmann, Konstanz, S. 225-256.

Farías, Ignacio (2013): Virtual attractors, actual assemblages: How Luhmann's theory of communication complements actor-network theory, in: European Journal of Social Theory 17, S. 24-41.

Foucault, Michel (1999): Die Ordnung der Dinge, Frankfurt / Main.

Fuchs, Peter (2004): Der Sinn der Beobachtung, Weilerswist.

Friedland, Roger / Robert R. Alford (1991): Bringing society back in, in: Walter Powell / Paul DiMaggio (Hrsg.), The New Institutionalism in Organizational Analysis, Chicago - London, S. 232-263.

Gad, Christopher / Casper B. Jensen (2010): On the consequences of post-ANT, in: Science, Technology \& Human Values 35, S. 55-80.

Garcia-Parpet, Marie-France (2007): The Social Construction of a Perfect Market, in: Donald A. MacKenzie / Fabian Muniesa / Lucia Siu (Hrsg.), Do Economists Make Markets? On the Performativity of Economics, Princeton / NJ, S. 21-53.

Garfinkel, Harold / Harvey Sacks (2004): Über formale Strukturen praktischer Handlungen, in: Jörg Strübing / Bernt Schnettler (Hrsg.), Methodologie interpretativer Sozialforschung. Klassische Grundlagentexte, Konstanz, S. 389-426.

Glaser, Barney G. / Anselm Strauss (1999): The Discovery of Grounded Theory: Strategies for Qualitative Research, New York / NY. 
Glaser, Barney G. (2002): Constructivist Grounded Theory?, in: Forum Qualitative Sozialforschung / Forum: Qualitative Social Research 3, abrufbar unter: http://www.qualitative-research.net/ index.php/fqs/article/view/825/1793, letztes Abrufdatum: 15.12.2014.

Günther, Gotthard (1963): Das Bewußtsein der Maschinen, Baden-Baden.

Günther, Gotthard (1976 a): Cybernetic Ontology and Tranjunctional Operations, in: Ders. (Hrsg.), Beiträge zur Grundlegung einer operationsfähigen Dialektik, Bd. 1, Hamburg, S. 249-328.

Günther, Gotthard (1976b): Das metaphysische Problem einer Formalisierung der transzendental-dialektischen Logik, in: Ders. (Hrsg.), Beiträge zur Grundlegung einer operationsfähigen Dialektik, Bd. 1, Hamburg, S. 189-247.

Günther, Gotthard (1976c): Die gebrochene Rationalität, in: Ders. (Hrsg.), Beiträge zur Grundlegung einer operationsfähigen Dialektik, Bd. 1, Hamburg, S. 115-140.

Günther, Gotthard (1978): Idee und Grundriss einer nicht-Aristotelischen Logik, Hamburg.

Günther, Gotthard (1979a): Die Theorie der „mehrwertigen“ Logik, in: Ders. (Hrsg.), Beiträge zur Grundlegung einer operationsfähigen Dialektik, Bd. 2, Hamburg, S. 181-202.

Günther, Gotthard (1979b): Life as Polycontexturality, in: Ders. (Hrsg.), Beiträge zur Grundlegung einer operationsfähigen Dialektik, Bd. 2, S. 283-306.

Günther, Gotthard (2000): Identität, Gegenidentität und Negativsprache, S. 1-60, abrufbar unter: http:// www.vordenker.de/ggphilosophy/gunther_identitaet.pdf, letztes Abrufdatum: 15.12.2014.

Harth, Jonathan (2014): Computergesteuerte Spielpartner. Formen der Medienpraxis zwischen Trivialität und Personalität, Wiesbaden.

Hegel, Georg W. F. (1999): Wissenschaft der Logik, Hamburg.

Heidegger, Martin (2006): Sein und Zeit, Tübingen.

Hirschauer, Stefan (2008): Die Empiriegeladenheit von Theorien und der Erfindungsreichtum der Praxis, in: Herbert Kalthoff / Stefan Hirschauer / Gesa Lindemann (Hrsg.), Theoretische Empirie: Zur Relevanz qualitativer Forschung, Frankfurt / Main, S. 165-187.

Holzinger, Markus (2013): Where are the missing practices?, in: Zeitschrift für Theoretische Soziologie 2, S. 31-55.

Jackson, Alecia Y. / Lisa A. Mazzei (2012): Thinking with Theory in Qualitative Research: Viewing Data across Multiple Perspectives, Abingdon / Oxfordshire - New York / NY.

Jansen, Till (2013): Mitbestimmung in Aufsichtsräten, Wiesbaden.

Jansen, Till (2014): Zweiwertigkeit und Mehrwertigkeit. Einige Vorschläge zu einer Soziologie der Polykontexturalität, in: Zeitschrift für theoretische Soziologie 3, S. 20-42.

Jansen, Till / Arist von Schlippe / Werner Vogd (2015): Kontexturanalyse - ein Vorschlag für rekonstruktive Sozialforschung in organisationalen Zusammenhängen, in: Forum Qualitative Sozialforschung / Forum: Qualitative Social Research 16, abrufbar unter: http://www.qualitative-research.net/ index.php/fqs/article/view/2198/3734, letztes Abrufdatum: 15.12.2014.

Jansen, Till / Werner Vogd (2013): Polykontexturale Verhältnisse - disjunkte Rationalitäten am Beispiel von Organisationen, in: Zeitschrift für theoretische Soziologie 2, S. 111-126.

Kaehr, Rudolf (1993): Disseminatorik: Zur Logik der ,Second Order Cybernetics‘. Von den ,Laws of Form` zur Logik der Reflexionsform, in: Dirk Baecker (Hrsg.), Kalkül der Form, Frankfurt / Main, S. 152-196.

Kalthoff, Herbert / Stefan Hirschauer / Gesa Lindemann (2008): Theoretische Empirie. Die Relevanz qualitativer Forschung, Frankfurt / Main.

Keller, Reiner (2014): Zukünfte der qualitativen Sozialforschung, in: Forum Qualitative Sozialforschung / Forum: Qualitative Social Research 15, abrufbar unter: http://www.qualitative-research.net/ index.php/fqs/article/view/2116/3633, letztes Abrufdatum: 15.12.2014. 
Knorr-Cetina, Karin (1989): Spielarten des Konstruktivismus. Einige Notizen und Anmerkungen, in: Soziale Welt 40, S. 86-96.

Knorr-Cetina, Karin (1992): Zur Unterkomplexität der Differenzierungstheorie. Empirische Anfragen an die Systemtheorie, in: Zeitschrift für Soziologie 21, S. 406-419.

Latour, Bruno (1987): Science in Action. How to Follow Scientists and Engineers Through Society, Cambridge / MA.

Latour, Bruno (1988): The Pasteurization of France, Cambridge / MA.

Latour, Bruno (2002): Wir sind nie modern gewesen. Versuch einer symmetrischen Anthropologie, Frankfurt / Main.

Latour, Bruno (2005): Reassembling the Social: An Introduction to Actor-Network-Theory, Oxford.

Latour, Bruno (2006): Über den Rückruf der ANT, in: Andréa Belliger / David J. Krieger (Hrsg.), ANThology. Ein einführendes Handbuch zur Akteur-Netzwerk-Theorie, Bielefeld, S. 561-572.

Latour, Bruno (2007): Eine neue Soziologie für eine neue Gesellschaft. Einführung in die Akteur-Netzwerk-Theorie, Frankfurt / Main.

Lindemann, Gesa (1999): Doppelte Kontingenz und reflexive Anthropologie, in: Zeitschrift für Soziologie 28, S. 165-181.

Lindemann, Gesa (2002): Die Grenzen des Sozialen. Zur sozio-technischen Konstruktion von Leben und Tod in der Intensivmedizin, München.

Lindemann, Gesa (2006): Die Du-Perspektive in der Hirnforschung, in: Jo Reichertz / Nadia Zaboura (Hrsg.), Akteur Gehirn - oder das vermeintliche Ende des handelnden Subjekts, Wiesbaden, S. 263-285.

Lindemann, Gesa (2008): „Allons enfants et faits de la patrie...“ Über Latours Sozial- und Gesellschaftstheorie sowie seinen Beitrag zur Rettung der Welt, in: Georg Kneer / Markus Schroer / Erhard Schüttpelz (Hrsg.), Bruno Latours Kollektive. Kontroversen zur Entgrenzung des Sozialen, Frankfurt / Main, S. 339-360.

Lindemann, Gesa (2010): Die Emergenzfunktion des Dritten - ihre Bedeutung für die Analyse der Ordnung einer funktional differenzierten Gesellschaft, in: Zeitschrift für Soziologie 39, S. 493-511.

Luhmann, Niklas (1996): Die Lebenswelt nach Rücksprache mit Phänomenologen, in: Gerhard Preyer / Georg Peter / Alexander Ulfig (Hrsg.), Protosoziologie im Kontext : „Lebenswelt“ und „System“ in Philosophie und Soziologie, Würzburg, S. 268-289.

Luhmann, Niklas (1997): Probleme mit operativer Schließung, Opladen.

Münch, Richard (1996): Autopoiesis per Definition, in: Gerhard Preyer / Georg Peter / Alexander Ulfig (Hrsg.), Protosoziologie im Kontext: „Lebenswelt“ und „System“ in Philosophie und Soziologie, Würzburg, S. 347-356.

Nassehi, Armin (2011): Gesellschaft der Gegenwarten, Studien zur Theorie der modernen Gesellschaft II, Frankfurt / Main.

Nassehi, Armin / Saake, Irmhild (2002): Kontingenz: Methodisch verhindert oder beobachtet? Ein Beitrag zur Methodologie der qualitativen Sozialforschung, in: Zeitschrift für Soziologie 31, S. 66-86.

Plessner, Helmuth (2003): Lachen und Weinen. Eine Untersuchung zu den Grenzen des menschlichen Verhaltens, in: Günter Dux / Odo Marquard / Elisabeth Ströker (Hrsg.), Ausdruck und menschliche Natur. Gesammelte Schriften VII, Frankfurt / Main, S. 201-389.

Popper, Karl R. (1994): Ausgangspunkte. Meine intellektuelle Entwicklung, Hamburg.

Rheinberger, Hans-Jörg (2006): Experimentalsysteme und epistemische Dinge, Frankfurt / Main.

Renn, Joachim (2006): Übersetzungsverhältnisse, Weilerswist.

Sayes, Edwin (2013): Actor-Network Theory and Methodology: Just What Does it Mean to Say that Nonhumans Have Agency?, in: Social Studies of Science 44, S. 134-149.

Stichweh, Rudolf (2000): Die Weltgesellschaft. Soziologische Analysen, Frankfurt / Main. 
Vogd, Werner (2007): Empirie oder Theorie? Systemtheoretische Forschung jenseits einer vermeintlichen Alternative, in: Soziale Welt 58, S. 295-321.

Vogd, Werner (2010): Gehirn und Gesellschaft, Weilerswist.

Vogd, Werner (2011): Systemtheorie und rekonstruktive Sozialforschung - eine Brücke, Opladen.

Vogd, Werner (2014 a): Problematische Selbstverhältnisse und Vermittlung. Qualitative Therapieforschung als Rekonstruktion der Reflexionsverhältnisse, in: KONTEXT (Zeitschrift für systemische Therapie und Familientherapie) 45, 7-22.

Vogd, Werner (2014b): Von der Physik zur Metaphysik - eine soziologische Rekonstruktion des Deutungsproblems der Quantentheorie, Weilerswist.

Wittgenstein, Ludwig (1992): Über Gewißheit. Werkausgabe Bd. 8, Frankfurt / Main.

Woolgar, Steve / Dorothy Pawluch (1985): Ontological Gerrymandering: The Anatomy of Social Problems Explanations, in: Social Problems 32, S. 214-227.

Dr. Till Jansen

Universität Witten / Herdecke

Lehrstuhl für Führung und Dynamik (WIFU)

Alfred-Herrhausen-Straße 50

58448 Witten

Till.Jansen@uni-wh.de

Prof. Dr. Werner Vogd

Universität Witten / Herdecke

Lehrstuhl für Soziologie

Alfred-Herrhausen-Straße 50

58448 Witten

Werner.Vogd@uni-wh.de 